\title{
Sustained Reentry in a 3D Regionally Ischemic Human Heart. A Simulation Study
}

\author{
Andres Mena Tobar ${ }^{1,2}$, Jose M Ferrero ${ }^{3}$, Jose F Rodriguez Matas ${ }^{1,4}$ \\ ${ }^{1}$ University of Zaragoza, Zaragoza, Spain \\ ${ }^{2}$ CIBER-BBN, Zaragoza, Spain \\ ${ }^{3}$ Politecnic University of Valencia, Valencia, Spain \\ ${ }^{4}$ Politecnico di Milano, Milan, Italy
}

\begin{abstract}
In this work, we have studied the vulnerable window and propagation patterns in a human heart during acute ischemia. A 3-D biventricular model of a human heart with realistic heterogeneity and fiber orientations has been considered. The ischemic region was located in the anterior left ventricular wall mimicking the occlusion of the circumflex artery. The electrical activity of the tissue was modeled with the monodomain model along with a modified version of the ten Tusscher 2006 ionic model. The model predicts the generation of sustained re-entrant activity in the form of a rotor around the ischemic zone. Patterns in the form of figure-of-eight were also observed within the vulnerable window. The re-entrant activity originates in the endocardial surface and propagates transmurally towards the epicardium.
\end{abstract}

\section{Introduction}

Ventricular tachycardia and fibrillation are known to be two types of cardiac arrhythmias that usually take place during acute ischemia and frequently lead to sudden death [1]. Even though these arrhythmias arise from different conditions, ischemia is the most important perpetrator among them. During ischemia, the delivery of nutrients to the myocardium diminishes, causing metabolic changes, which result in a progressive deterioration of the electric activity in the injured region [2]. These metabolic changes are mainly hypoxia, increased concentrations of the extracellular potassium $\left[\mathrm{K}^{+}\right]_{\mathrm{o}}$ (hyperkalemia), decrease of intracellular ATP, and acidosis [3]. From an electrophysiological point of view, these metabolic changes imply alterations in the action potential (AP), excitability, conduction velocity (CV), and effective refractive period (ERP) among others, creating a substrate for arrhythmias and fibrillation $[3,4]$. In addition, the impact of ischemia in the myocardium is characterized by a high degree of heterogeneity both intramurally and transmurally. In the tissue affected by acute ischemia, two zones can be distinguished: i) the central ischemic zone (CIZ) corresponding to the core of the tissue suffering from the lack of blood, and ii) a border zone (BZ) which comprises changes in electrophysiological properties between the healthy and ischemic regions [5].

In this work, we have studied the different propagation patterns and determined the vulnerable window in a human heart during acute ischemia.

\section{Methods}

The electrical activity of the heart was modelled by means of the well established monodomain equation [6]

$$
\begin{gathered}
\boldsymbol{\nabla} \cdot(\mathbf{D} \nabla V)=C_{m} \frac{\partial V}{\partial t}+J_{i o n}(V, \mathbf{u})+J_{s t m} \\
\frac{\partial \mathbf{u}}{d t}=\mathbf{f}(\mathbf{u}, V, t)
\end{gathered}
$$

where $V$ is the transmembrane potential, $\mathbf{D}$ is the second order anisotropic conductivity tensor, $C_{m}$ the membrane capacitance, $J_{i o n}$ the ionic current, $J_{\text {stm }}$ the stimulus current, and $\mathrm{u}$ is a set of state variables associated with the ionic model. The set of equations (1) is subject to the zero flux boundary conditions.

$$
\mathbf{n} \cdot(\mathbf{D V V})=0 .
$$

In this work, the electric activity of the cell was modelled using the tenTusscher model (TP06) [7]. In simulating ischemia, the value of the parameters affected by ischemia were set to the experimental values corresponding to approximately ten minutes of ischemia. Hypoxia was considered by incorporating the ATPsensitive $\mathrm{K}^{+}$current $\left(\mathrm{I}_{\mathrm{K}(\mathrm{ATP})}\right)$ to the TP06 model. The formulation proposed by Ferrero et al [8] was adopted, and which formulation is given as

$$
I_{K(A T P)}=g_{0}\left(\frac{\left[K^{+}\right]_{o}}{5.4}\right)^{0.24} f_{M} f_{T} f_{N} f_{A T P}\left(V-E_{K}\right),
$$


where $g_{0}$ is the maximum macroscopic channel conductance, $f_{M}, f_{N}, f_{T}$ are $\mathrm{Mg}^{2+}, \mathrm{Na}^{+}$and temperaturedependant correction factors, $f_{A T P}$ is the fraction of activated channels, $V$ the transmembrane potential, and $E_{K}$ is the reversal potential of the channel. The maximum channel macroscopic conductance and the fraction of activated channels, $f_{A T P}$, have been modified with respect to its original formulation

$$
\begin{gathered}
f_{A T P}=\frac{1}{1+\left(\frac{[A T P]_{i}}{K_{m}}\right)^{H}}, \\
K_{m}=\alpha\left(35.8+17.9[A D P]_{i}{ }^{0.256}\right), \\
H=1.3+4.44 e^{-0.09[A D P]_{i}},
\end{gathered}
$$

where $[A T P]_{i}$ and $[A D P]_{i}$ are the intracellular ATP and ADP concentrations in $\mu \mathrm{m} / \mathrm{L}$ respectively. Parameters $\alpha$, and $\mathrm{g}_{0}$ where identified by fitting experimental data available for different animal models. Table 1 summarizes the identified parameters for the different cell types.

Table 1. Value of the parameters for the $\mathrm{I}_{\mathrm{K}(\mathrm{ATP})}$ current adapted to the TP06 model

\begin{tabular}{ccccc}
\hline Cell Type & $g_{0},(\mathrm{mS})$ & $\alpha$ & $\begin{array}{c}\square \square(\mathrm{ms}) \\
\text { control }\end{array}$ & $\begin{array}{c}\text { APD(ms) } \\
\text { Ischemic }\end{array}$ \\
\hline EPI & 4.5 & 1.0 & 299 & 265 \\
ENDO & 4.5 & 0.32 & 393 & 178 \\
MID & 4.5 & 0.86 & 298 & 141 \\
\hline
\end{tabular}

The model of the acute ischemic heart followed closely that proposed in [9]. The ischemic region was composed of realistically dimensioned transitional border zones (BZ), a normal zone (NZ), and the central zone (CZ) of ischemia. In the $\mathrm{CZ},\left[\mathrm{K}^{+}\right]_{\mathrm{o}}$ was set to $9.9 \mathrm{mM}[5,10]$, the inward $\mathrm{Na}^{+}$and L-type $\mathrm{Ca}^{2+}$ currents were scaled by a factor of 0.85 to imitate acidosis $[11,12]$, and $[A T P]_{i}$ and $[A D P]_{i}$ concentrations were set to $5 \mathrm{mM}$ and $99 \mu \mathrm{M}$ respectively [13]. Table 1 gives the AP duration (APD) obtained with the modified model in control and ischemic conditions. In addition, a $1.0 \mathrm{~mm}$ wash-out zone in the endocardium was incorporated as proposed in Wilensky et al. [14].

The ischemic region was located in the anterior side of the left ventricle mimicking the occlusion of the circumflex artery. Realistic cell heterogeneity and fiber anisotropy were considered in the model. Figure 1a shows the finite element model of the human heart and a detail of the considered ischemic region.

The model was stimulated at a frequency of $1.25 \mathrm{~Hz}$, at normal stimulation points (see Figure $1 \mathrm{~b}$ ), for 45 seconds, for preconditioning, followed by an extra-stimulus located in the subendocardial border zone [13] (see Figure $1 c)$, with different coupling intervals $(\mathrm{CI})$. The reentrant patterns were studied for five seconds after the ectopic stimulus under two conditions: i) the basic stimulation at the normal activation points interrupted after the ectopic beat, ii) the basic stimulation at the normal activation points was continued after the ectopic beat.

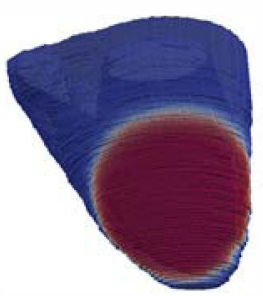

(a)

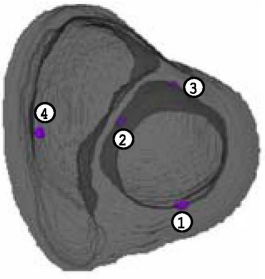

(b)

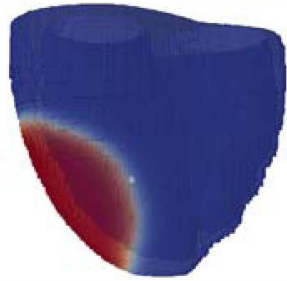

(c)
Figure 1. The computational model a) Detail of the ischemic zone, b) location of the normal stimulation sites, c) location of the ectopic stimulation.

Computations were performed on a GPU Tesla M2090 (6GRAM DDR5) with the software TOR [15] using the operator splitting and a semi-implicit scheme with a fixed time step of $0.02 \mathrm{~ms}$.

\section{Results}

Results show spatial heterogeneities in the propagated action potential, as reported experimentally, throughout the regional ischemic tissue, such as resting membrane potential $(-85.2 \mathrm{mV}$ in $\mathrm{NZ}$, and $-72.5 \mathrm{mV}$ in the $\mathrm{CZ}$, with potentials varying between these values in the BZ). During a basic beat, activity spreads from two directions into the ischemic area, whereas after the premature beat, activity spreads from the right side of the border into the ischemic area as shown in Figure 2.

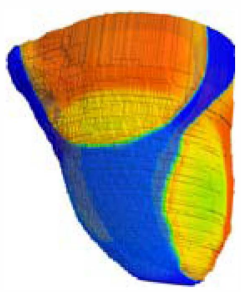

(a)

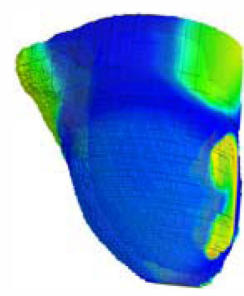

(b)
Figure 2. Depolarization pattern through the ischemic area during basic activation (left panel), and right after the ectopic beat (right panel).

For CIs in the range $265-300 \mathrm{~ms}$, reentry occurred in the heart. In addition, within this window, the reentrant pattern was not altered by the presence of normal sinus rhythm stimulation. For CIs bellow $265 \mathrm{~ms}$, double blocked occurred without generating re-entrant activity. On the contrary, for CI's larger than $300 \mathrm{~ms}$, conduction through the ischemic zone occurred. Within the vulnerable window, re-entrant activity originated in the endocardium as a single rotor, that propagated transmurally toward the epicardium as shown in Figure 3 for $\mathrm{CI}=270 \mathrm{~ms}$ and $\mathrm{CI}=290 \mathrm{~ms}$. 


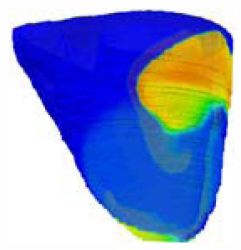

(a)

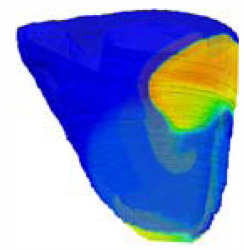

(b)
Figure 3. Reentrant front in the ischemic heart a) $\mathrm{CI}=270$ $\mathrm{ms}$ b) $\mathrm{CI}=290 \mathrm{~ms}$.

Even though the reentrant activity always started in the endocardium in the form of a rotor, the pattern of the reentrant wavefront, even though always limited to the ischemic region, changed depending on the CI. For CIs between $270 \mathrm{~ms}$ and $285 \mathrm{~ms}$, reentrant patterns consisting of a single rotor and figure of eight were observed as shown in the sequence of pictures in Figure 4 for $\mathrm{CI}=270$ ms. In the instant shown, the pattern changed from a single rotor, to a figure of eight, to then return to a single rotor. The reentry was always observed within the ischemic region.
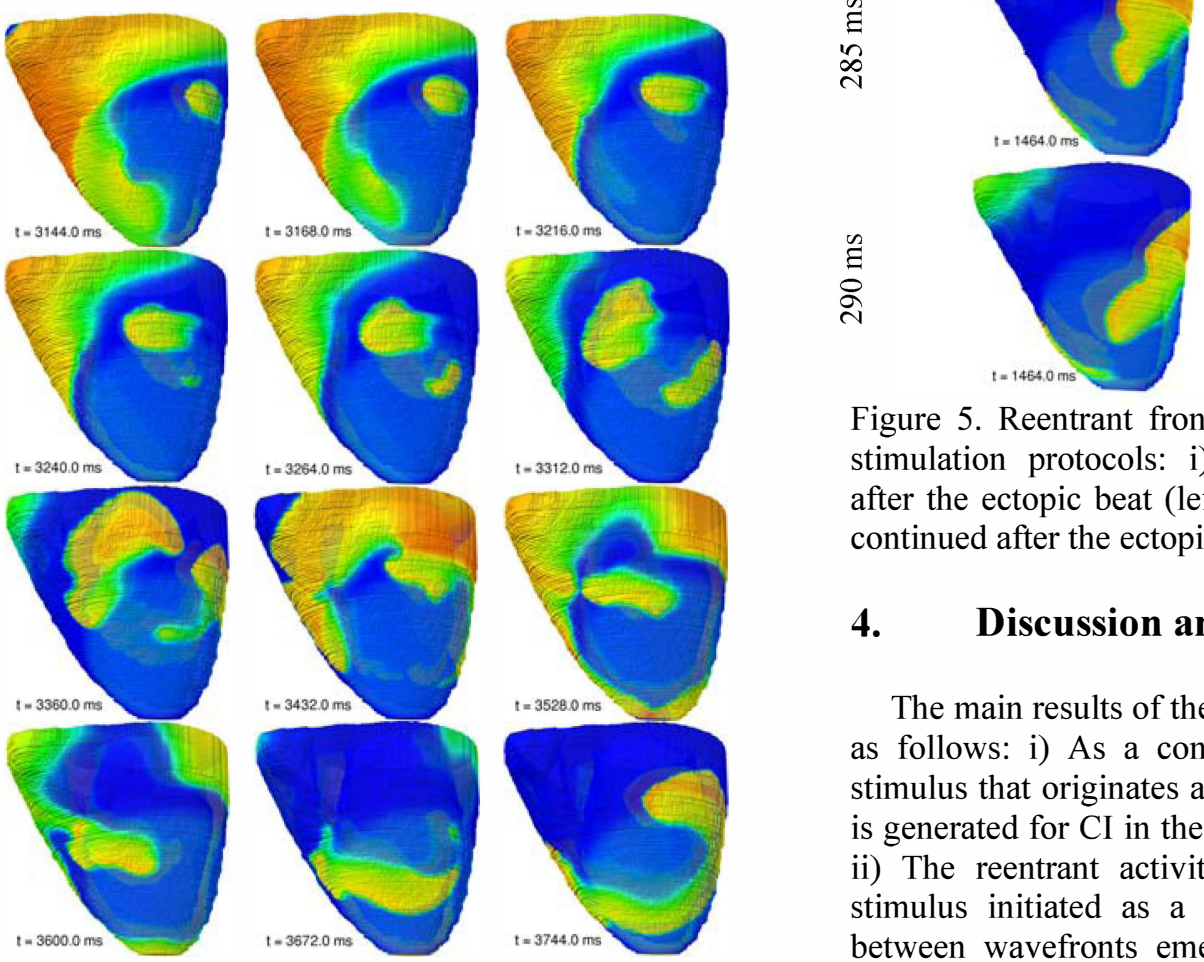

Figure 5. Reentrant front for different CIs and the two stimulation protocols: i) Basic stimulation interrupted after the ectopic beat (left column), ii) Basic stimulation continued after the ectopic beat (right column).

\section{Discussion and conclusions}

The main results of the simulations can be summarized as follows: i) As a consequence of the applied extrastimulus that originates an ectopic beat, reentrant activity is generated for CI in the range between 265 and $300 \mathrm{~ms}$; ii) The reentrant activity generated due to the extrastimulus initiated as a consequence of the interaction between wavefronts emerging from the wash-out zone into the ischemic zone.

During the reentrant activity, one circuit (single rotor) of fairly large dimension was basically observed, i.e., the activity circles around an area of block the size of the ischemic area. For some CI's, double circuits were observed but not completed (or sustained), and the reentrant activity continued because of one single reentrant circuit. These patterns from our simulations has been reported in the experimental work by Janse et al [13] in pig and dog hearts. For instance, Figure 8 in ref [13] 
shows single circuit and the eight shape reentrant pattern also obtained in our simulations (see Figure 2).

However, differently to the experimental studies by Janse et al., none of the cases studied lead to ventricular fibrillation. In all our simulations the reentrant activity was always restricted to the ischemic zone with clearly defined reentry circuits.

We have only monitored the reentrant activity up to five seconds during which we have observed perpetuating activity for a restricted vulnerable window. In some cases, i.e., $\mathrm{CI}=265 \mathrm{~ms}$ and $\mathrm{CI}=300 \mathrm{~ms}$, the reentrant activity was spontaneously terminated after 3 seconds of activity, or before five seconds. This type of behavior was also reported in [13] where tachycardia terminated within $30 \mathrm{sec}$ after initiation. Additional studies on larger ischemic zones are required in order to determine if the size of the ischemic area may favor the onset of ventricular fibrillation in the ischemic heart. In addition, the location of the ectopic activity maybe important for both, the size of the vulnerable window and the reentrant pattern.

In conclusion, the model predicts the generation of reentry within the ischemic zone due to the heterogeneity in the refractory period between the ischemic affected area and the normal myocardium. The observed patterns obtained with the simulations are in good agreement with experimental studies conducted in pig and dog hearts subjected to acute regional ischemia.

\section{Acknowledgements}

This project was partially supported by the "VI Plan Nacional de Investigación Científica, Desarrollo e Innovación Tecnológica" from Ministerio de Economía y Competitividad of Spain (grant numbers TIN2012-37546C03-01 and TIN2012-37546-C03-03) and the European Commission (European Regional Development Funds ERDF - FEDER)

\section{References}

[1] Rubart M, Zipes DP. Mechanisms of sudden cardiac death. J Clin Invest 2005; 115(9):2305-2315.

[2] Katz A. Physiology of the Heart. Lippincott Williams and Wilkins 2001.

[3] Carmeliet E. Cardiac ionic currents and acute ischemia: from channels to arrhythmias. Physiol Rev 1999; 79: 9171017.

[4] Janse MJ, Kleber AG. Electrophysiological changes and ventricular arrhythmias in the early phase of regional myocardial ischemia. Circ Res 1981; 49(5): 1069-1081.

[5] Coronel R. Heterogeneity in extracellular potassium concentration during early myocardial ischaemia and reperfusion: implications for arrhythmogenesis. Cardiovasc. Res. 1994; 28(6):770-777.

[6] Geselowitz DB, Miller III, WT. A bidomain model for anisotropic cardiac muscle. Ann Biomed Eng 1983;
$11: 315-334$.

[7] ten Tusscher KHWJ, Panfilov AV. Alternants and spiral breakup in a human ventricular tissue model. Am J Physiol Heart Circ Physiol 2006; 291: H1088-H1100.

[8] Ferrero JM, Jr., Saiz J, Ferrero JM, Thakor NV. Simulation of action potentials from metabolically impaired cardiac myocytes. Role of ATP-sensitive K+ current. Circ. Res. 1996; 79(2):208-221.

[9] Heidenreich E, Ferrero JM, Rodriguez JF. Modeling the Human under Acute Ischemia. In: B. Calvo, E. Peña (eds.), Patient-Specific Computational Modeling, Lecture Notes in Computational Vision and Biomechanics 5 $\square$. Dordrecht: Springer 2012: 81-104.

[10] Coronel R, Fiolet JW, Wilms-Schopman FJ, Schaapherder AF, Johnson TA, Gettes LS, Janse MJ. Distribution of extracellular potassium and its relation to electrophysiologic changes during acute myocardial ischemia in the isolated perfused porcine heart. Circ. 1988; 77(5):1125-1138.

[11] Ferrero JM, Trenor B, Rodriguez B, Saiz J. Electrical activity and reentry during acute regional myocardial ischemia: insights from simulations. International Journal of Bifurcation and Chaos 2003; 13: 3703-3715.

[12] Yatani A, Brown AM, Akaike N. Effect of extracellular $\mathrm{pH}$ on sodium current in isolated, single rat ventricular cells. J. Membr. Biol. 1984; 78(2):163-168.

[13] Janse MJ, van Capelle FJ, Morsink H, Kleber AG, WilmsSchopman F, Cardinal R, d'Alnoncourt C, Durrer D. Flow of "injury" current and patterns of excitation during early ventricular arrhythmias in acute regional myocardial ischemia in isolated porcine and canine hearts. Evidence for two different arrhythmogenic mechanisms. Circ Res 1980; 47(2): 151-165.

[14] Wilensky RL, Tranum-Jensen J, Coronel R, Wilde AAM, Fiolet JWT, Janse MJ. Subendocardial border zone during acute ischemia of rabbit heart: an electrophysiologic, metabolic, and morphologic correlative study. Circulation 1986; 74: 1137-1146.

[15] Mena Tobar A, Ferrero JM, Rodriguez JF. GPU accelerated solver for nonlinear reaction-diffusion systems. Application to the electrophysiology problem. Comp Phys Comm 2015; doi: 10.1016/j.cpc.2015.06.018.

\section{Address for correspondence}

José F. Rodríguez

Departamento de Ingeniería Mecánica (Grupo AMB)

Universidad de Zaragoza

c/ María de Luna 3,

50018 Zaragoza, Spain

jfrodrig@unizar.es

Dipartimento de Chimica, Materiali e Ingegneria Chimica "Giulio Nata"

Politecnico di Milano

Piazza Leonardo da Vinci 32,

20113 Milan, Italy

josefelix.rodriguezmatas@polimi.it 